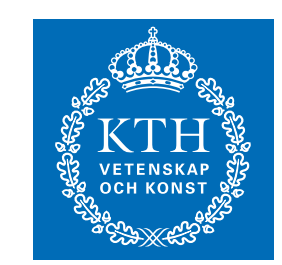

KTH Electrical Engineering

\title{
On the Principles of Multicell Precoding with Centralized and Distributed Cooperation
}

\author{
Best Paper Award \\ International Conference on Wireless Communications and Signal Processing
} (WCSP 2009)

November 13 - 15, Nanjing, China, 2009

(C) 2009 IEEE. Published in the 2009 International Conference on Wireless Communications and Signal Processing (WCSP 2009), scheduled for November 13

- 15, Nanjing, China. Personal use of this material is permitted. However, permission to reprint/republish this material for advertising or promotional purposes or for creating new collective works for resale or redistribution to servers or lists, or to reuse any copyrighted component of this work in other works, must be obtained from the IEEE. Contact: Manager, Copyrights and Permissions /

IEEE Service Center / 445 Hoes Lane / P.O. Box 1331 / Piscataway, NJ 08855-1331, USA. Telephone: + Intl. 908-562-3966.

\section{EMIL BJÖRNSON AND BJÖRN OTTERSTEN}

\section{Stockholm 2009}

\author{
KTH Royal Institute of Technology \\ ACCESS Linnaeus Center \\ Signal Processing Lab
}

DOI: $10.1109 /$ WCSP.2009.5371760

KTH Report: IR-EE-SB 2009:044 


\title{
On the Principles of Multicell Precoding with Centralized and Distributed Cooperation
}

\author{
(Invited Paper)
}

\author{
Emil Björnson \\ ACCESS Linnaeus Center \\ Royal Institute of Technology (KTH) \\ SE-100 44 Stockholm, Sweden \\ Email: emil.bjornson@ee.kth.se
}

\author{
Björn Ottersten \\ ACCESS Linnaeus Center \\ Royal Institute of Technology (KTH) \\ SE-100 44 Stockholm, Sweden \\ Email: bjorn.ottersten@ee.kth.se
}

\begin{abstract}
Cooperative precoding is an attractive way of improving the performance in multicell downlink scenarios. By serving each terminal through multiple surrounding base stations, inter-cell interference can be coordinated and higher spectral efficiency achieved, especially for terminals at cell edges. The optimal performance of multicell precoding is well-known as it can be treated as a single cell with distributed antennas. However, the requirements on backhaul signaling and computational power scales rapidly in large and dense networks, which often makes such fully centralized approaches impractical. In this paper, we review and generalize some recent work on multicell precoding with both centralized and distributed cooperation. We propose practical precoding strategies under Rician channel conditions, and illustrate how the major gain of multicell precoding originates from having good base station synchronization and not from making centralized precoding decisions.
\end{abstract}

\section{INTRODUCTION}

To achieve higher data rates in wireless communications, it is crucial to enhance the spectral efficiency without increasing the demands on bandwidth and transmit power. For this purpose, multiantenna techniques are attractive since they can provide performance that increases with the number of antennas when other radio resources are fixed [1]. In the multiuser downlink, these performance gains can be achieved through simultaneous transmissions to multiple terminals [1] [4]. Channel state information (CSI) is required to separate these transmissions spatially and to control the co-user interference. Accurate CSI is often the bottleneck that limits the downlink performance. Thus, resources need to be spent on learning and analyzing channels for transmission design [5].

An attractive feature of cellular systems is that each base station can optimize its cell throughput in a decentralized manner. To fully exploit the multiantenna gain, base station cooperation is however required, otherwise the inter-cell interference fundamentally limits the performance of terminals at cell edges [6]. A high-speed backhaul network can be used to facilitate cooperation, but this infrastructure is costly, causes delays, and computational demands increase rapidly as more information is jointly processed. Thus, there is great interest in

B. Ottersten is also with securityandtrust.lu, University of Luxembourg. The research leading to these results has received funding from the European Research Council under the European Community's Seventh Framework Programme (FP7/2007-2013) / ERC Grant Agreement No. 228044. partially or fully distributed forms of cooperation that achieve reliable interference control by limited backhaul signaling and thus are scalable to large cellular networks [7], [8].

Multicell precoding represents the scenario when multiple base stations serve each terminal, and its sum capacity and capacity region with perfect CSI and various power constraints were derived in [9] and [10], respectively. The impact of multicell precoding on cell edge terminals was studied in [11] and [12] under simplified models where only two singleantenna base stations affect each terminal. Under general channel conditions, fully centralized schemes were discussed in [13] and a zero-forcing (ZF) scheme with centralized power allocation was proposed in [14]. The achievable rate region with beamforming was characterized in [15], [15] and the optimal beamformers were shown to be linear combinations of maximum ratio transmission (MRT) and ZF. Fully distributed schemes were recently proposed and analyzed in [15]-[17] based on a virtual signal-to-interference-and-noise (SINR) framework that combines the strengths of MRT and ZF.

In this paper, we consider multicell precoding (also known as network MIMO) under Rician channel conditions. Practical precoding strategies are discussed for both centralized and distributed cooperation. The main contributions are:

- We derive expressions for the expected sum rate in multicell precoding systems with Rician CSI and discuss how it can be used for centralized precoding design.

- The virtual SINR framework in [15]-[17] is extended for distributed beamforming design under Rician CSI. A simple power allocation scheme is proposed.

- The performance of centralized and distributed precoding is evaluated numerically. We illustrate the performance difference between centralized and distributed schemes and show the importance of base station synchronization.

\section{SySTEM MOdEL}

We consider a communication scenario with $K_{t}$ transmitters (e.g., base stations in a cellular system) equipped with $N_{t} \geq K_{r}$ antennas each and $K_{r}$ single-antenna receivers. The transmitters and receivers are denoted $\mathrm{BS}_{j}$ and $\mathrm{MS}_{k}$, respectively, for $j \in\left\{1, \ldots, K_{t}\right\}$ and $k \in\left\{1, \ldots, K_{r}\right\}$. 


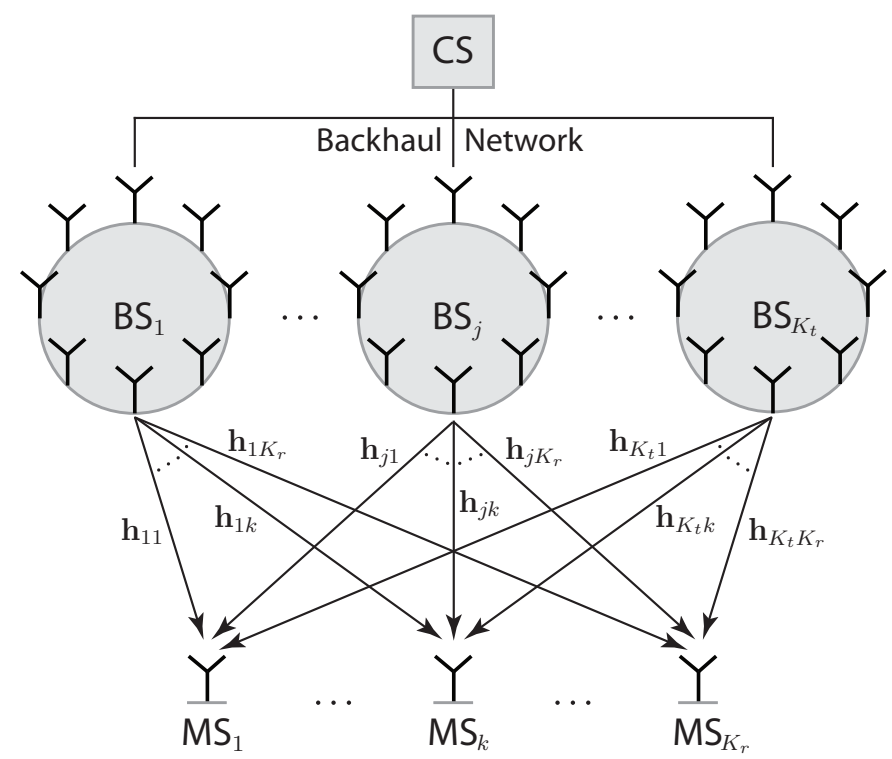

Fig. 1. The basic scenario with $K_{t}$ base stations transmitting to $K_{r}$ terminals (illustrated for $N_{t}=8$ transmit antennas). The base stations can cooperate through a backhaul network, supervised by a central station.

The transmitters are connected to a backhaul network that is controlled by a central station (CS); see Fig. 1 .

Let $\mathbf{x}_{j} \in \mathbb{C}^{N_{t}}$ be the signal transmitted by $\mathrm{BS}_{j}$ and let the received signal at $\mathrm{MS}_{k}$ be denoted by $y_{k} \in \mathbb{C}$. The propagation channel to $\mathrm{MS}_{k}$ is assumed to be narrowband, flat fading with the system model

$$
y_{k}=\sum_{j=1}^{K_{t}} \mathbf{h}_{j k}^{H} \mathbf{x}_{j}+n_{k},
$$

where $\mathbf{h}_{j k} \in \mathbb{C}^{N_{t}}$ represents the channel between $\mathrm{BS}_{j}$ and $\mathrm{MS}_{k}$ and $n_{k} \in \mathcal{C N}\left(0, \sigma^{2}\right)$ is noise. $\mathcal{C N}(\cdot, \cdot)$ denotes a circular symmetric complex Gaussian vector. To emulate practical systems, transmitters and receivers have different CSI:

- Receive-side CSI: $\mathrm{MS}_{k}$ has full local CSI, which means perfect estimates of $\mathbf{h}_{j k}$, for $j=1, \ldots, K_{t}$, and knowledge of the noise power $\sigma^{2}$.

- Transmit-side CSI: $\mathrm{BS}_{j}$ has partial local CSI, which means a known distribution $\mathbf{h}_{j k} \in \mathcal{C N}\left(\overline{\mathbf{h}}_{j k}, \mathbf{Q}_{j k}\right)$ for $k=1, \ldots, K_{r}$ and of the noise power $\sigma^{2}$.

Many different types of transmit-side CSI can be jointly modeled in this manner, for example

1) Perfect Instantaneous CSI: $\mathbf{h}_{j k}=\overline{\mathbf{h}}_{j k}$ (i.e., $\mathbf{Q}_{j k}=\mathbf{0}$ );

2) Estimated CSI: $\overline{\mathbf{h}}_{j k}$ is a channel estimate with error covariance $\mathbf{Q}_{j k}$ (see [5] for details on estimation);

3) Statistical CSI: Long-term statistical CSI of either a Rician $\left(\overline{\mathbf{h}}_{j k} \neq \mathbf{0}\right)$ or Rayleigh $\left(\overline{\mathbf{h}}_{j k}=\mathbf{0}\right)$ fading channel. Observe that in these cases, the transmitters are only assumed to have CSI that can be obtained locally, for example using reverse-link estimation in time-division duplex (TDD) systems or feedback in frequency-division duplex (FDD) systems.

\section{A. Cooperative Multicell Precoding}

The data symbol $s_{k} \in \mathcal{C N}(0,1)$ is intended for $\mathrm{MS}_{k}$ and is assumed to be available at all transmitters. This enables mul- ticell precoding, where each receiver is served simultaneously by multiple transmitters. In the analysis, perfect base station synchronization is assumed, while synchronization errors will be studied in Section IV. Under the assumption of linear precoding, the signal transmitted by $\mathrm{BS}_{j}$ becomes in general

$$
\mathbf{x}_{j}=\sum_{k=1}^{K_{r}} \sqrt{p_{j k}} \mathbf{w}_{j k} s_{k}
$$

where beamformers $\mathbf{w}_{j k}$ have unit norms (i.e., $\left\|\mathbf{w}_{j k}\right\|=1$ ) and $p_{j k}$ represents the power allocated for transmission to $\mathrm{MS}_{k}$. The transmitter $\mathrm{BS}_{j}$ is subject to an individual average power constraint of $P_{j}$; that is, $\mathbb{E}\left\{\left\|\mathbf{x}_{j}\right\|^{2}\right\}=\sum_{k=1}^{K_{r}} p_{j k} \leq P_{j}$.

When the receivers treat co-user interference as noise, the instantaneous SINR at $\mathrm{MS}_{k}$ can be expressed as

$$
\mathrm{SINR}_{k}=\frac{\left|\sum_{j=1}^{K_{t}} \sqrt{p_{j k}} \mathbf{h}_{j k}^{H} \mathbf{w}_{j k}\right|^{2}}{\sum_{\substack{k=1 \\ \bar{k} \neq k}}^{K_{r}}\left|\sum_{j=1}^{K_{t}} \sqrt{p_{j \bar{k}}} \mathbf{h}_{j k}^{H} \mathbf{w}_{j \bar{k}}\right|^{2}+\sigma^{2}}=\frac{\left|a_{k k}\right|^{2}}{\sum_{\substack{k=1 \\ \bar{k} \neq k}}^{K_{r}}\left|a_{\bar{k} k}\right|^{2}+\sigma^{2}}
$$

using the notation $\mathbf{a}_{k} \triangleq\left[a_{1 k} \ldots a_{K_{r} k}\right]^{H} \in \mathcal{C N}\left(\mathbf{b}_{k}, \mathbf{S}_{k}\right)$ with

$$
\begin{gathered}
\mathbf{b}_{k} \triangleq \sum_{j=1}^{K_{t}} \mathbf{W}_{j}^{H} \overline{\mathbf{h}}_{j k}, \quad \mathbf{S}_{k} \triangleq \sum_{j=1}^{K_{t}} \mathbf{W}_{j}^{H} \mathbf{Q}_{j k} \mathbf{W}_{j}, \\
\text { and } \mathbf{W}_{j} \triangleq\left[\sqrt{p_{j 1}} \mathbf{w}_{j 1} \ldots \sqrt{p_{j K_{r}}} \mathbf{w}_{j K_{r}}\right] .
\end{gathered}
$$

For later use, we define the vector of elements in the denominator $\widetilde{\mathbf{a}}_{k} \triangleq\left[a_{1 k} \ldots a_{k-1 k} a_{k+1 k} \ldots a_{K_{r} k}\right]^{H} \in \mathcal{C N}\left(\widetilde{\mathbf{b}}_{k}, \widetilde{\mathbf{S}}_{k}\right)$, where $\widetilde{\mathbf{b}}_{k}$ is achieved by removing the $k$ th element of $\mathbf{b}_{k}$ and $\widetilde{\mathbf{S}}_{k}$ by removing $k$ th column and $k$ th row of $\mathbf{S}_{k}$.

This SINR characterization requires that all pairs of channel vectors $\left(\mathbf{h}_{j k}, \mathbf{h}_{\bar{j} k}\right)$ are statistically independent for all $k$ and $j \neq \bar{j}$. In addition, only transmit-side CSI (global or local) can be used in the precoding design, as in our scenario. Next, the characterization in (3) will be used to derive the expected sum rate and to analyze different precoding schemes.

\section{Centralized And Distributed Precoding}

The performance of the considered communication system evidently depends on the precoding; that is, the beamformers $\mathbf{w}_{j k}$ and the power allocation coefficients $p_{j k}$. Herein, the performance measure is the expected sum rate and thus we would like to find the precoding that solves

$$
\max _{\substack{\left\{\mathbf{w}_{j k} \in \mathbb{C}^{N_{t}} \\\left\{p_{j k} \geq 0 \forall j, k ;\left\|\mathbf{w}_{j k}\right\|=1\right\}\right.}} \sum_{k=1}^{K_{r}} \mathbb{E}\left\{\log _{2}\left(1+\operatorname{SINR}_{k}\right)\right\} .
$$

For given beamformers and power allocation coefficients, the expected sum rate can be calculated using the following theorem. To simplify the notation, we first introduce the function $g(\cdot, \cdot, \cdot)$ that for example can be used to calculate the expected rate for interference-free transmission over channel $\mathbf{h}_{j k}: g\left(\overline{\mathbf{h}}_{j k}, \mathbf{Q}_{j k}, \sigma^{2}\right)=\mathbb{E}\left\{\log _{2}\left(1+\left\|\mathbf{h}_{j k}\right\|^{2} / \sigma^{2}\right)\right\}$.

Lemma 1. Let $\overline{\mathbf{v}}=\left[v_{1} \ldots v_{N}\right]^{T}$ and $\boldsymbol{\Lambda}=\operatorname{diag}\left(\lambda_{1}, \ldots, \lambda_{N}\right)$, where $\lambda_{i} \neq \lambda_{j}>0$ for all $i \neq j$. For $\mathbf{v} \in \mathcal{C N}(\overline{\mathbf{v}}, \boldsymbol{\Lambda})$ and $\sigma^{2}>0$, 
we define the function $g\left(\overline{\mathbf{v}}, \boldsymbol{\Lambda}, \sigma^{2}\right) \triangleq \mathbb{E}\left\{\log _{2}\left(1+\|\mathbf{v}\|^{2} / \sigma^{2}\right)\right\}$ that can be calculated as

$$
\begin{aligned}
g\left(\overline{\mathbf{v}}, \boldsymbol{\Lambda}, \sigma^{2}\right)= & \frac{e^{-\overline{\mathbf{v}}^{H} \boldsymbol{\Lambda}^{-1} \overline{\mathbf{v}}}}{\log (2)} \sum_{i=1}^{N} \frac{e^{\sigma^{2} / \lambda_{i}}}{\prod_{j \neq i}\left(1-\frac{\lambda_{j}}{\lambda_{i}}\right)} \sum_{m=0}^{\infty} d_{i}[m] \\
& \times \sum_{k=0}^{\infty} \frac{\left(\frac{\left|v_{i}\right|^{2}}{\lambda_{i}}\right)^{k}}{(m+k) !} \sum_{j=0}^{k} \frac{\left(-\frac{\sigma^{2}}{\lambda_{i}}\right)^{j}}{j !} \sum_{l=0}^{k-j} \frac{\Gamma\left(l, \frac{\sigma^{2}}{\lambda_{i}}\right)}{\Gamma(l+1)} .
\end{aligned}
$$

Here, $\Gamma(\cdot)$ and $\Gamma(\cdot, \cdot)$ denotes the Gamma function and the upper incomplete Gamma function, respectively, and

$$
\begin{aligned}
& d_{i}[m] \triangleq \underset{j \neq i}{\left.\underset{j}{*} d_{i j}\right)}[m] \text { for } \\
& d_{i j} \triangleq e^{\gamma_{j i}} \gamma_{i j} L_{m}\left(-\gamma_{j i}\right) u[m] \text { and } \gamma_{i j} \triangleq \frac{\left|\bar{v}_{i}\right|^{2}}{\lambda_{i}\left(1-\frac{\lambda_{i}}{\lambda_{j}}\right)}
\end{aligned}
$$

where $\star$ is discrete convolution, $L_{m}(\cdot)$ the $m$ th order Laguerre polynomial and $u[m]=1$ for $m \geq 0$ and zero otherwise.

Proof: The proof is given in the appendix.

Using the SINR characterization in (3), Lemma 1 gives the following expression for the expected sum rate.

Theorem 1. Let $\mathbf{S}_{k}=\mathbf{U}_{k} \boldsymbol{\Lambda}_{k} \mathbf{U}_{k}^{H}$ and $\widetilde{\mathbf{S}}_{k}=\widetilde{\mathbf{U}}_{k} \widetilde{\boldsymbol{\Lambda}}_{k} \widetilde{\mathbf{U}}_{k}^{H}$ define eigenvalue decompositions, for all $k$. Then, the expected sum rate can be calculated as ${ }^{1}$

$$
\sum_{k=1}^{K_{r}} \mathbb{E}\left\{\log _{2}\left(1+\operatorname{SINR}_{k}\right)\right\}=\sum_{k=1}^{K_{r}} g\left(\overline{\mathbf{v}}_{k}, \boldsymbol{\Lambda}_{k}, \sigma^{2}\right)-g\left(\widetilde{\mathbf{v}}_{k}, \widetilde{\boldsymbol{\Lambda}}_{k}, \sigma^{2}\right)
$$

where $\overline{\mathbf{v}}_{k}=\mathbf{U}_{k}^{H} \mathbf{b}_{k}$ and $\widetilde{\mathbf{v}}_{k}=\widetilde{\mathbf{U}}_{k}^{H} \widetilde{\mathbf{b}}_{k}$.

Proof: For each receiver, the expected rate is

$$
\begin{aligned}
& \mathbb{E}\left\{\log _{2}\left(1+\operatorname{SINR}_{k}\right)\right\} \\
& \quad=\mathbb{E}\left\{\log _{2}\left(\sigma^{2}+\left\|\mathbf{a}_{k}\right\|^{2}\right)\right\}-\mathbb{E}\left\{\log _{2}\left(\sigma^{2}+\left\|\widetilde{\mathbf{a}}_{k}\right\|^{2}\right)\right\} .
\end{aligned}
$$

Since the Euclidean norm is invariant under unitary transformations, $\left\|\mathbf{a}_{k}\right\|^{2}=\left\|\mathbf{U}_{k}^{H} \mathbf{a}_{k}\right\|^{2}$ with $\mathbf{U}_{k}^{H} \mathbf{a}_{k} \in \mathcal{C N}\left(\overline{\mathbf{v}}_{k}, \boldsymbol{\Lambda}_{k}\right)$ and we can apply Lemma 1 to evaluate the first term of (8). The second term is evaluated in the corresponding way.

Next, we discuss how to maximize the expected sum rate with either centralized or distributed cooperation.

\section{A. Fully and Partially Centralized Cooperation}

In a system with centralized cooperation, all base stations share CSI. Thus, the central station is sufficiently informed to calculate the expected sum rate in Theorem 1. However, the expression for $g(\cdot, \cdot, \cdot)$ in (6) contains two infinite summations that need approximations. Fortunately, these originate from [18, Theorem 9] and were derived to be suitable for truncation. Let $M_{\max }$ and $K_{\max }$ denote the truncated upper limits of $\sum_{m=0}^{\infty}$ and $\sum_{k=0}^{\infty}$ in (6), respectively. Fig. 2 shows the relative error when computing $g\left(\overline{\mathbf{v}}_{1}, \boldsymbol{\Lambda}_{1}, 1\right)$, where

\footnotetext{
${ }^{1}$ For notational convenience, the theorem is formulated as if $\mathbf{S}_{k}$ and $\tilde{\mathbf{S}}_{k}$ have full rank, for all $k$. This will not hold in the special case of perfect zero-forcing beamforming, but the corresponding expected sum rate can be determined by removing the zero-valued eigenvalues from $\boldsymbol{\Lambda}_{k}$ and $\tilde{\boldsymbol{\Lambda}}_{k}$ (and incorporating the corresponding rows of $\overline{\mathbf{v}}_{k}$ and $\widetilde{\overline{\mathbf{v}}}_{k}$ into the $\sigma^{2}$-terms).
}

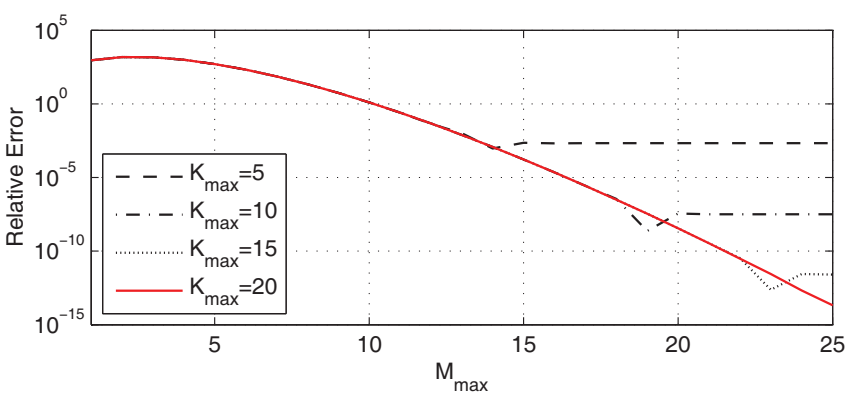

Fig. 2. Relative error when calculating $g\left(\overline{\mathbf{v}}_{1}, \boldsymbol{\Lambda}_{1}, 1\right)$ by truncating the two infinite sums in Lemma 1 to $M_{\max }+1$ and $K_{\max }+1$ terms, respectively.

$\overline{\mathbf{v}}_{1}=\left[e^{i \pi / 4} e^{i 3 \pi / 4} e^{i 5 \pi / 4} e^{i 7 \pi / 4}\right]^{T}, \boldsymbol{\Lambda}_{1}=\operatorname{diag}(0.5,1,1.5,2)$, and the value with $M_{\max }=K_{\max }=50$ is used as the exact value. We only need $M_{\max }=15$ and $K_{\max }=10$ to reach a relative error of about $10^{-4}$. Observe that $K_{\max }$ has a limited impact on the error, which is good since the computational complexity scales faster with $K_{\max }$ than with $M_{\max }$.

By truncation, the expected sum rate can be evaluated with reasonable complexity. The precoding design can then be solved by standard multidimensional optimization procedures [19], but the optimal solution can be hard to obtain since the problem is non-convex (even for perfect instantaneous CSI). In addition, the dimension and complexity scales rapidly with the number of terminals, which makes the demands on the central station unreasonable in many practical systems. Most of the complexity lies in the selection of beamforming vectors and therefore [13], [14] considered heuristic beamforming based on ZF (these beamformers can be calculated using local CSI) combined with optimized power allocation. In the case of Rician CSI, we generalize ZF beamforming as follows.

Strategy 1. Generalized zero-forcing $(G-Z F)$ is achieved by $\mathbf{w}_{j k}^{(\mathrm{G}-\mathrm{ZF})}=\frac{\mathbf{v}_{j k}}{\left\|\mathbf{v}_{j k}\right\|}$ for all $j, k$, where $\mathbf{v}_{j k}$ is the dominating eigenvector of $\boldsymbol{\Pi}_{\mathcal{S}_{j k}}^{\perp}\left(\mathbf{Q}_{j k}+\overline{\mathbf{h}}_{j k} \overline{\mathbf{h}}_{j k}^{H}\right) \boldsymbol{\Pi}_{\mathcal{S}_{j k}}^{\perp}$.

Here, $\boldsymbol{\Pi}_{\mathcal{S}_{j k}}^{\perp}$ is the orthogonal projection matrix onto the complement of $\mathcal{S}_{j k}=\operatorname{span}\left(\bigcup_{\bar{k} \neq k}\left\{\mathbf{w}_{j \bar{k}}^{(\mathrm{G}-\mathrm{MRT})}\right\}\right)$, where $\mathbf{w}_{j \bar{k}}^{(\mathrm{G}-\mathrm{MRT})}$ is the dominating eigenvector of $\left(\mathbf{Q}_{j \bar{k}}+\overline{\mathbf{h}}_{j \bar{k}} \overline{\mathbf{h}}_{j \bar{k}}^{H}\right)$.

When heuristic beamforming is applied, the precoding design reduces to a rather simple power allocation problem that requires global CSI. An iterative solution was proposed in [14] for outage minimization and heuristic sum rate optimizing schemes were given in [17] for different types of CSI. To reflect the potential performance of future research, we use numerically optimized power allocation in Section IV.

\section{B. Fully Distributed Cooperation}

The practical difficulties of distributing CSI over the backhaul network and high complexity of joint precoding design motivates the analysis of fully distributed cooperation. Next, we will propose heuristic beamforming and power allocation strategies that can be computed locally using only local CSI.

In general, the goal of beamforming is to maximize the signal power at the intended terminal while minimizing the interference caused at other terminals [2]. These ambitions are counteracting and represented by MRT (good at low SNR) and 
ZF (good at high SNR), respectively. Recently, it was shown that all weighted sum rate maximizing beamformers are linear combinations of MRT and ZF [15], [17] (under instantaneous or zero-mean statistical CSI). This result is substantial for achieving effective and robust distributed precoding, since the MRT and ZF vectors can be calculated locally.

In other words, selecting effective beamformers corresponds to finding the right balance between maximizing the signal power and minimizing the interference. For interference channels (ICs) where only one base station serves each terminal, this balancing act can be solved optimally by maximizing the ratio between signal power and interference caused at other terminals plus noise [20]. In our multicell precoding scenario, this approach is suboptimal but our numerical examples will show that it still can perform well. The following so-called layered virtual SINR strategy is an extension to the multicell strategies proposed in [15]-[17] to the case of Rician CSI.

Strategy 2. Layered Virtual SINR (LVSINR) maximizing beamformers are achieved by, for all $j, k$, solving

$$
\mathbf{w}_{j k}^{(\mathrm{LVSINR})}=\underset{\|\mathbf{w}\|=1}{\arg \max } \frac{\mathbb{E}\left\{\left|\mathbf{h}_{j k}^{H} \mathbf{w}\right|^{2}\right\}}{\frac{\sigma^{2}}{p_{j k}}+\sum_{\bar{k} \neq k} \mathbb{E}\left\{\left|\mathbf{h}_{j \bar{k}}^{H} \mathbf{w}\right|^{2}\right\}} .
$$

Observe that $\mathbb{E}\left\{\left|\mathbf{h}_{j \bar{k}}^{H} \mathbf{w}\right|^{2}\right\}=\mathbf{w}^{H}\left(\mathbf{Q}_{j \bar{k}}+\overline{\mathbf{h}}_{j \bar{k}} \overline{\mathbf{h}}_{j \bar{k}}^{H}\right) \mathbf{w}$ and that the optimization in (9) is a generalized Rayleigh quotient that can be solved by eigenvalue techniques; $\mathbf{w}_{j k}^{\text {(LVSINR) }}$ is the dominating eigenvector of $\mathbf{C}_{j k}^{1 / 2}\left(\mathbf{Q}_{j k}+\overline{\mathbf{h}}_{j k} \overline{\mathbf{h}}_{j k}^{H}\right) \mathbf{C}_{j k}^{1 / 2}$, where $\mathbf{C}_{j k} \triangleq \frac{\sigma^{2}}{p_{j k}} \mathbf{I}+\sum_{\bar{k} \neq k}\left(\mathbf{Q}_{j \bar{k}}+\overline{\mathbf{h}}_{j \bar{k}} \overline{\mathbf{h}}_{j \bar{k}}^{H}\right)$. Note that the solution to (9) is non-unique, since $\mathbf{w} e^{j \theta}$ yields the same function value for any $\theta \in \mathbb{R}$. By selecting the solution that makes the inner product $\overline{\mathbf{h}}_{j k}^{H} \mathbf{w}$ positive and real-valued, the signals arriving along the mean components from different transmitters will add up constructively (assuming proper synchronization).

Observe that the beamforming vectors in Strategy 2 depend on the power allocation coefficients. We propose the following heuristic power allocation scheme, which generalizes the schemes proposed in [16] to the case of Rician CSI.

Strategy 3. A distributed power allocation is achieved by

$$
p_{j k}^{\text {heuristic }}=\frac{\operatorname{tr}\left\{\mathbf{Q}_{j k}+\overline{\mathbf{h}}_{j k} \overline{\mathbf{h}}_{j k}^{H}\right\}}{\sum_{\bar{k}=1}^{K_{r}} \operatorname{tr}\left\{\mathbf{Q}_{j \bar{k}}+\overline{\mathbf{h}}_{j \bar{k}} \overline{\mathbf{h}}_{j \bar{k}}^{H}\right\}} P_{j} .
$$

This power allocation scheme follows the intuition of allocating more power to strong terminals, since weak terminals hopefully are served more effectively by other base stations.

\section{NumericAl EXAMPLES}

In this section, we illustrate the precoding performance in a scenario with $K_{t}=2$ base stations with $N_{t}=3$ antennas, and $K_{r}=2$ terminals located at the cell edge between the base stations. We will compare centralized and distributed schemes, and investigate how mismatches in the transmission synchronization affect the performance. These errors can for example be due to estimation uncertainty, hardware delays, clock drifts, and minor channel changes. We emulate these

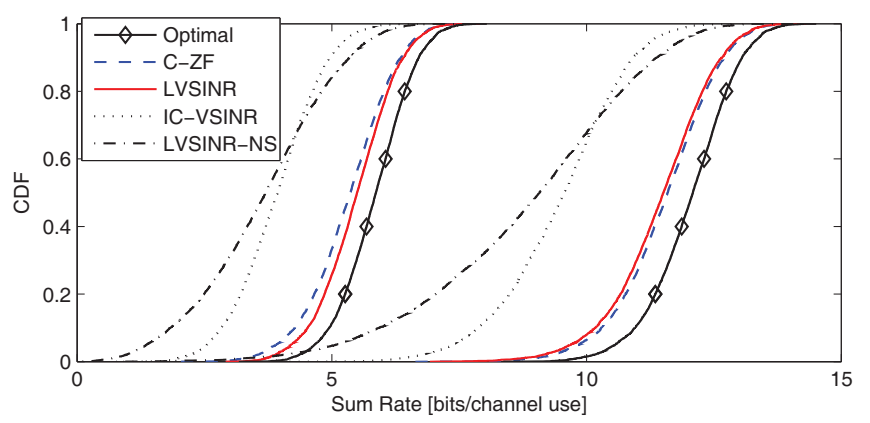

Fig. 3. CDFs of sum rates with multicell precoding in a scenario with $K_{t}=K_{r}=2, N_{t}=3$, instantaneous CSI, and terminals at the cell edge.

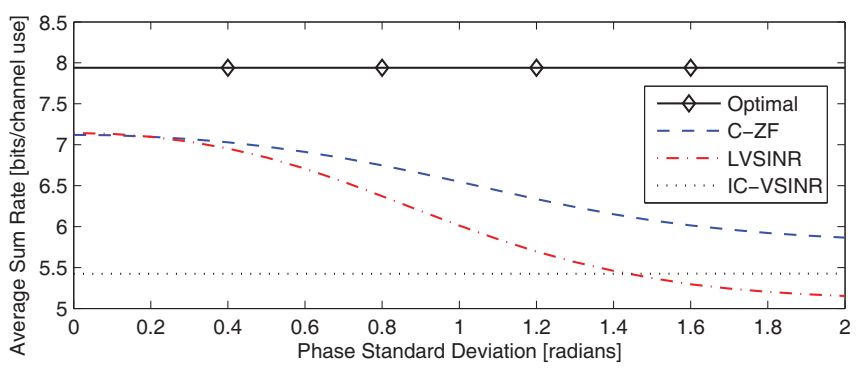

Fig. 4. Average sum rate as a function of standard phase deviation $\sigma_{\phi}$. The actual channels are modeled as $\mathbf{h}_{j k}^{\text {actual }}=\mathbf{h}_{j k} e^{i \phi_{j k}}$, where $\phi_{j k} \in \mathcal{N}\left(0, \sigma_{\phi}^{2}\right)$.

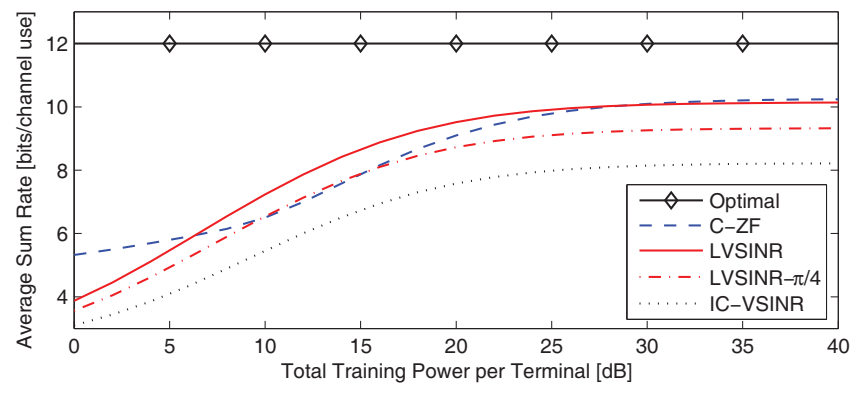

Fig. 5. Average sum rate (at $10 \mathrm{~dB}$ SNR) as a function of the total training power spent on reverse-link channel estimation per terminal.

mismatches by letting the actual channel be $\mathbf{h}_{j k}^{\text {actual }}=\mathbf{h}_{j k} e^{i \phi_{j k}}$, where the phase deviation $\phi_{j k}$ is unknown.

In Fig. 3, we give the cumulative distribution functions (CDFs) of the sum rate when all channel vectors are known (i.e., $\mathbf{Q}_{j k}=\mathbf{0}$ for all $j, k$ ) and generated as i.i.d. Rayleigh fading. The performance is given for an average SNR of either 0 or $10 \mathrm{~dB}$ (when allocating all power to one terminal). Sum rate maximizing precoding is compared with $\mathrm{ZF}$ in Strategy 1 with optimal centralized power allocation (C-ZF), distributed LVSINR beamforming in Strategy 2 with power allocation in Strategy 3, and the IC-VSINR approach in [20]. Interestingly, the performance loss when comparing the distributed LVSINR strategy with optimal precoding is only 5-8 percent. In addition, the gap disappears when the optimal beamforming is replaced by ZF. Thus, it seems like having global CSI is only needed for optimal precoding and not for practical schemes.

Thus, we argue that the major gain of multicell precoding is not related to having global CSI, but depends on having perfect base station synchronization. In Fig. 3, LVSINR-NS represents the case with no synchronization (i.e., $\phi_{j k} \in U[-\pi, \pi)$ ). 
We observe a significant performance loss compared with perfect synchronization, and the point of multicell precoding is ruined as the interference channel approach (IC-VSINR) yields better performance. The transition between perfect and poor synchronization is further analyzed in Fig. 4 (at $5 \mathrm{~dB}$ SNR). Here, the phase synchronization error is modeled as $\phi_{j k} \in$ $\mathcal{N}\left(0, \sigma_{\phi}^{2}\right)$ and the resulting average sum rate is displayed for varying standard deviation $\sigma_{\phi}$. While the IC-VSINR approach in [20] is independent of synchronization errors (since one base station serves each terminal), the centralized $\mathrm{ZF}$ and distributed LVSINR approaches show gradual performance degradations. The ZF scheme will however stabilize on higher performance than the LVSINR scheme, since its centralized power allocation can switch to serving each terminal only by the base station with the strongest link.

Finally, we study the impact of channel estimation errors. Fig. 5 shows the performance (at $10 \mathrm{~dB}$ SNR) with reverselink estimation (in TDD mode [5]) with varying total training power (per terminal). ZF with optimal power allocation (CZF), distributed LVSINR, and IC-VSINR (all with perfect synchronization) are compared and show a similar dependence on the estimation quality that stabilize around a total training power of $20 \mathrm{~dB}$. At medium training power, the LVSINR scheme is more resilient towards estimation errors. We also show the LVSINR approach with $\phi_{j k} \in \mathcal{N}\left(0,\left(\frac{\pi}{4}\right)^{2}\right)$ and observe that the synchronization error leads to a simple translation towards lower performance.

\section{CONCLUSION}

Cooperative multicell precoding has the potential of increasing the spectral efficiency by having multiple base stations serving each terminal. We have derived an expression for the expected sum rate, under Rician CSI, that can be used for centralized precoding optimization with global CSI. In practice, the backhaul signaling and computational complexity required to derive the optimal precoding is often prohibitive. Fortunately, heuristic beamforming strategies like ZF and LVSINR show very good performance, using only local CSI. Based on simulations, we argue that the gain of centralized precoding can be far below the hardware requirements for achieving this solution. When perfect transmission synchronization cannot be guaranteed, the performance of multicell precoding schemes will degrade, but centralized power allocation and scheduling with (limited) global CSI can be used to control the loss.

\section{APPENDIX}

Proof of Lemma 1: The PDF of $\|\mathbf{v}\|^{2} \geq 0$ was given in [18, Theorem 9] as a series expansion suitable for implementation:

$$
f(\rho)=\sum_{i=1}^{N} \frac{e^{-\overline{\mathbf{v}}^{H} \boldsymbol{\Lambda}^{-1} \overline{\mathbf{v}}} e^{-\rho / \lambda_{i}}}{\lambda_{i} \prod_{j \neq i}\left(1-\frac{\lambda_{j}}{\lambda_{i}}\right)} \sum_{m=0}^{\infty} \frac{d_{i}[m] I_{m}\left(2 \frac{\left|\bar{v}_{i}\right| \sqrt{\rho}}{\lambda_{i}}\right)}{\left(\frac{\left|\bar{v}_{i}\right| \sqrt{\rho}}{\lambda_{i}}\right)^{m}},
$$

where $I_{m}(\cdot)$ is the $m$ th modified Bessel function of the first kind. Then, the expected value can be calculated as

$$
\mathbb{E}\left\{\log _{2}\left(1+\frac{\|\mathbf{v}\|^{2}}{\sigma^{2}}\right)\right\}=\int_{0}^{\infty} \log _{2}\left(1+\frac{\rho}{\sigma^{2}}\right) f(\rho) d \rho .
$$

By using the expansion $\left(\left|\bar{v}_{i}\right| \sqrt{\rho} / \lambda_{i}\right)^{-m} I_{m}\left(2\left|\bar{v}_{i}\right| \sqrt{\rho} / \lambda_{i}\right)=$ $\sum_{k=0}^{\infty}\left(\left|\bar{v}_{i}\right|^{2} \rho / \lambda_{i}^{2}\right)^{k} /(k !(m+k) !)$, the part that contains $\rho$ is

$$
\begin{aligned}
\int_{0}^{\infty} \log _{2}\left(1+\frac{\rho}{\sigma^{2}}\right) e^{-\rho / \lambda_{i}}\left(\rho / \lambda_{i}\right)^{k} d \rho \\
=\frac{\lambda_{i} e^{\sigma^{2} / \lambda_{i}} k !}{\log (2)} \sum_{j=0}^{k} \frac{\left(-\frac{\sigma^{2}}{\lambda_{i}}\right)^{j}}{j !} \sum_{l=0}^{k-j} \frac{\Gamma\left(l, \frac{\sigma^{2}}{\lambda_{i}}\right)}{\Gamma(l+1)},
\end{aligned}
$$

where the equality follows from a change of variables $(\tilde{\rho}=$ $\left.1+\rho / \sigma^{2}\right)$ and straightforward integration. Using the Bessel function expansion, substitution of (12) into (11) gives (6).

\section{ACKNOWLEDGMENT}

The authors would like to acknowledge useful discussions with Randa Zakhour and David Gesbert, EURECOM.

\section{REFERENCES}

[1] M. Sharif and B. Hassibi, "On the capacity of MIMO broadcast channels with partial side information," IEEE Trans. Inf. Theory, vol. 51, 2005.

[2] D. Gesbert, M. Kountouris, R. Heath, C.-B. Chae, and T. Sälzer, "Shifting the MIMO paradigm," IEEE Signal Process. Mag., 2007.

[3] D. Love, R. Heath, V. Lau, D. Gesbert, B. Rao, and M. Andrews, "An overview of limited feedback in wireless communication systems," IEEE J. Sel. Areas Commun., vol. 26, no. 8, pp. 1341-1365, 2008.

[4] E. Björnson, D. Hammarwall, and B. Ottersten, "Exploiting quantized channel norm feedback through conditional statistics in arbitrarily correlated MIMO systems," IEEE Trans. Signal Process., vol. 57, 2009.

[5] E. Björnson and B. Ottersten, "A framework for training-based estimation in arbitrarily correlated Rician MIMO channels with Rician disturbance," IEEE Trans. Signal Process., submitted for publication.

[6] S. Venkatesan, A. Lozano, and R. Valenzuela, "Network MIMO: Overcoming intercell interference in indoor wireless systems," in Proc. IEEE ACSSC'07, 2007, pp. 83-87.

[7] P. Marsch and G. Fettweis, "On multicell cooperative transmission in backhaul-constrained cellular systems," Ann. Telecommun., 2008.

[8] B. L. Ng, J. Evans, S. Hanly, and D. Aktas, "Distributed downlink beamforming with cooperative base stations," IEEE Trans. Inf. Theory, vol. 54, pp. 5491-5499, 2008.

[9] W. Yu, "Uplink-downlink duality via minimax duality," IEEE Trans. Inf. Theory, vol. 52, pp. 361-374, 2006.

[10] H. Weingarten, Y. Steinberg, and S. Shamai, "The capacity region of the Gaussian multiple-input multiple-output broadcast channel," IEEE Trans. Inf. Theory, vol. 52, pp. 3936-3964, 2006.

[11] O. Somekh, B. Zaidel, and S. Shamai, "Sum rate characterization of joint multiple cell-site processing," IEEE Trans. Inf. Theory, 2007.

[12] S. Jing, D. Tse, J. Soriaga, J. Hou, J. Smee, and R. Padovani, "Multicell downlink capacity with coordinated processing," EURASIP J. Wirel. Commun. Netw., 2008.

[13] M. Karakayali, G. Foschini, and R. Valenzuela, "Network coordination for spectrally efficient communications in cellular systems," IEEE Wireless Commun. Mag., vol. 13, pp. 56-61, 2006.

[14] M. Kobayashi, M. Debbah, and J. Belfiore, "Outage efficient strategies in network MIMO with partial CSIT," in Proc. IEEE ISIT'09, 2009.

[15] E. Björnson, R. Zakhour, D. Gesbert, and B. Ottersten, "Distributed multicell and multiantenna precoding: Characterization and performance evaluation," in Proc. IEEE GLOBECOM'09, 2009.

[16] R. Zakhour and D. Gesbert, "Distributed multicell-MIMO precoding using the layered virtual SINR framework," in IEEE Trans. Wireless Commun., submitted for publication.

[17] E. Björnson, R. Zakhour, D. Gesbert, and B. Ottersten, "Cooperative multicell precoding: Rate region characterization and distributed strategies with instantaneous and statistical CSI," IEEE Trans. Signal Process., submitted for publication.

[18] D. Hammarwall, M. Bengtsson, and B. Ottersten, "Acquiring partial CSI for spatially selective transmission by instantaneous channel norm feedback," IEEE Trans. Signal Process., vol. 56, pp. 1188-1204, 2008.

[19] S. Boyd and L. Vandenberghe, Convex Optimization. Cambridge University Press, 2004.

[20] R. Zakhour and D. Gesbert, "Coordination on the MISO interference channel using the virtual SINR framework," in Proc. WSA'09, 2009. 